\title{
On-Line non-intrusive Monitoring of Particulate Solid Materials in Gas Flowlines Using Acoustic Sensor and ML Techniques
}

\author{
${ }^{1}$ Kuda Tijjani Aminu and ${ }^{2}$ Don McGlinchey \\ ${ }^{1}$ Department of Engineering, Abubakar Tatari Ali Polytechnic, Bauchi, Nigeria \\ ${ }^{2}$ Department of Engineering, Glasgow Caledonian University, United Kingdom
}

\begin{abstract}
This paper describes initial steps towards developing a real-time quantitative particulate solids' (sand) monitoring system for Multiphase flowlines based on acoustic monitoring and machine learning techniques. The concentration and the velocity of the solids were varied during experimental trials. A conventional contact microphone mounted externally to a production flowline bend was used for recording the emitted acoustic signal. Features extracted from the signal were used as input to Time Delay Neural Network (TDNN) with solids concentration and velocity label to a training set. The TDNN achieved low values of normalised root mean square error (NRMSE) for all the models compared to the classical neural network.
\end{abstract}

Keywords: Multiphase flow, Acoustic Monitoring, Machine Learning, Signal processing, Condition Monitoring.

\section{INTRODUCTION}

The presence of sand in hydrocarbon flowline presents a technical challenge crucial to the operators within the petroleum industry[1-2], which has led to considerable research interest in acoustic monitoring of flowlines [3-5], the work presented in this paper describes early attempts to build on the lesson learned in the oil and gas industry where a nonintrusive, real-time, on-line measurement of sand concentration and velocity is required. Acoustic signal processing with integrated machine learning is believed to have the potential to meet this requirement. In order to effectively process the acoustic signal, among other techniques, feature representation of audio signals previously developed for music and speech processing applications were used to describe the evolution of the signals in the time and frequency domains.

Nevertheless, the sand grains produced during oil and gas production often get transported to the surface alongside the produced hydrocarbon and consequently impinge upon the wall of the production flowline, particularly at flow restriction zones where the flow changes direction, namely a bend [6]. These impingements tend to create stress waves that are captured as acoustic signals by the acoustic sensor mounted externally to the bend. In recent years, these signals have been used in monitoring various industrial processes, such as condition monitoring [7-8], on-line particle sizing [9-13], slurry flow[12-13]. This paper proposes an approach incorporating an acoustic sensor, signal processing techniques and machine learning algorithm to realise real-time measurement of the flow characteristics of particle-laden multiphase flow. Put simply, the acoustic sensor is employed for its simplicity, nonintrusiveness and low cost.

\section{MATERIALS AND METHOD}

\subsection{Experimental Setup}

A series of experimental tests were carried out using the GCU multiphase flow loop test facility shown in Figure1. The pipeline layout in the loop was of mild steel internal diameter roughly $50 \mathrm{~mm}$, with total length of around $12 \mathrm{~m}$. The majority of the pipeline layout was in the horizontal plane and included four 90 degree standard radius bends. The acoustic recording sensor was installed at the bend. The tests were carried out with flow conditions having sand concentrations in the order of 3 to 44 by mass. 


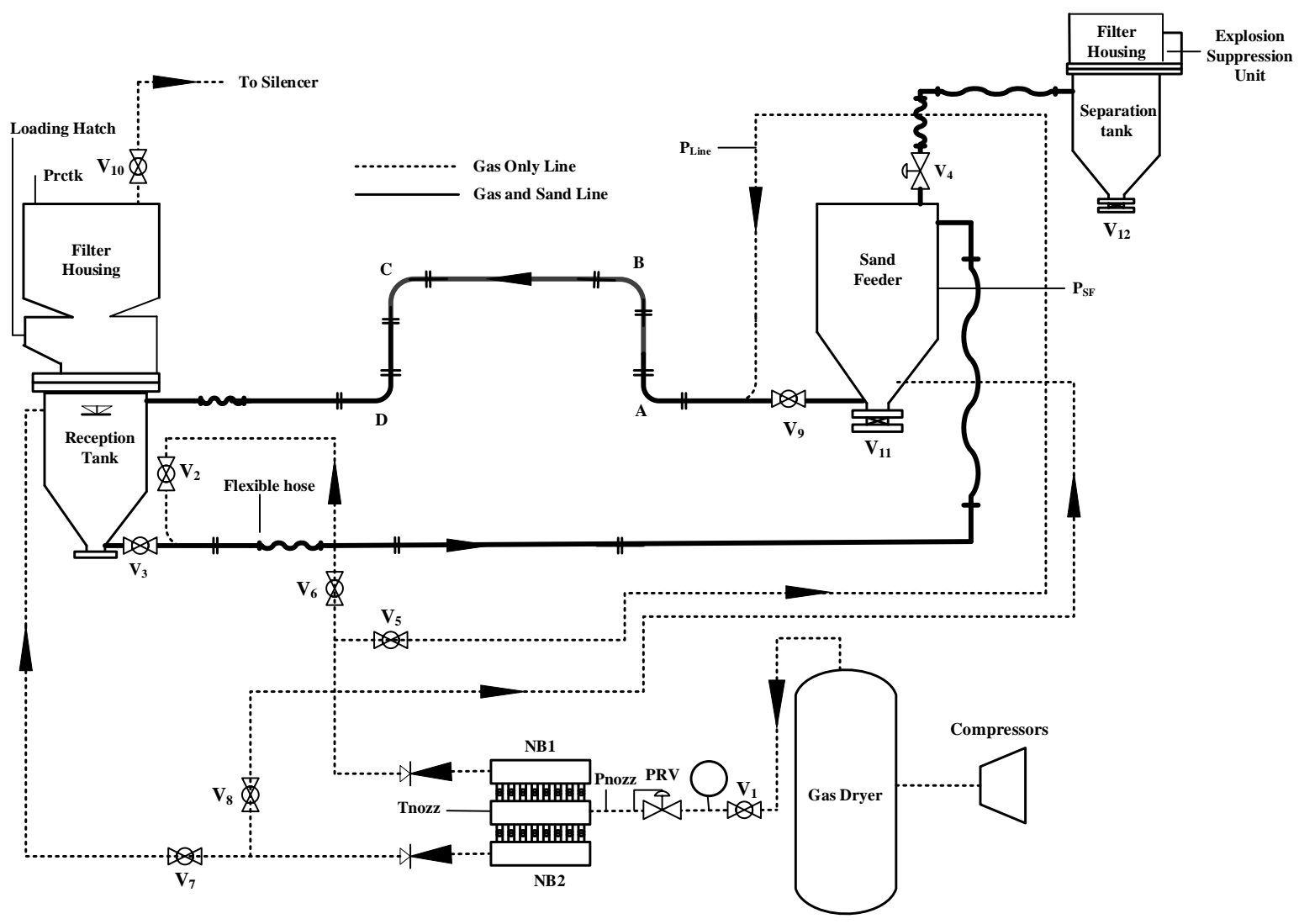

Figure 1- Multiphase flow loop schematic diagram

The flow of gas through the sand feeder and the transport line was achieved using a nozzle bank and an upstream pressure control valve. The rate of flow of sand by mass was measured using the change in mass signals from the load cells installed at the reception tank. The location of the acoustic sensor is illustrated in Figure 2. The sand used for the experiments is the Garside 2EW sand. This sand has an average particle size of $360.18 \mu \mathrm{m}$ and its size distribution spread between $60 \mu \mathrm{m}$ and $2000 \mu \mathrm{m}$. The transporting gas used is a compressed air supplied by two screw compressors. The compressors were capable of delivering approximately $0.123 \mathrm{~m}^{3} / \mathrm{s}$ of free air with maximum steady pressure of around $7.5 \mathrm{barg}$

\subsection{Data Acquisition Setup}

The signals emitted by different sand concentrations contribute to the acoustics employed in the preprocessing stage. These signals have been conditioned by a signal conditioning circuit and thus have sufficient signal strength. The complete setup consists of: four contact microphones mounted on the four bends; four single ended pressure transducers to record the pressure at various locations within the layout; a temperature sensor mounted upstream to record the gas temperature; three load cells installed on the reception tank to measure the solid mass flow rate; national instruments USB-6211 and USB-6212 data acquisition systems - UBS-6212 for the microphones and USB-6211 for the other sensors; HP laptop with National Instruments Signal Express software.

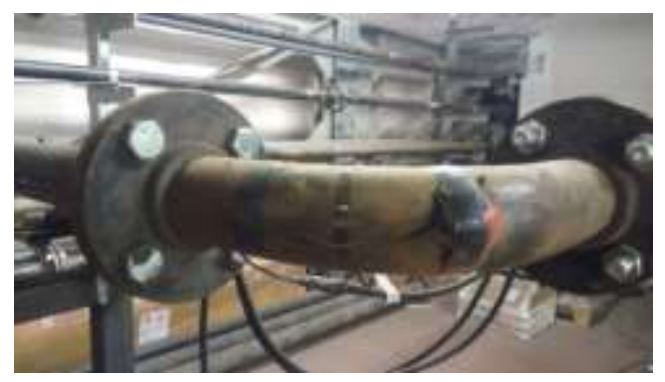

Figure 2 - Location of the Acoustic Sensor

The microphone location presented in Figure 2 was chosen based on the assumption that the sand particles provide the highest kinetic energy at the bend location compared to the straight section of the flowline. Data was collected at a rate of $44100 \mathrm{~Hz}$. 


\subsection{Measurement flow characteristic parameters}

The procedure implemented to measure the sand concentration (SC) and gas velocity is discussed in this section. Sand concentration is determined using Equation (1). In Equation (1), $\dot{m}_{s}$ represents the sand mass flow rate whereas $\dot{m}_{g}$ simply represents the total gas mass flow rate.

$$
S C=\frac{\dot{m}_{s}}{\dot{m}_{g}}
$$

However, the sand flow rate is calculated from the test data at steady line pressure using Equation (2)

$$
\dot{m}_{s}=\frac{\text { sand mass Collected }(\mathrm{kg})}{\text { Time }(\mathrm{s})}
$$

From Equation (3), the total gas mass flow $\dot{m}_{g}$ can be determined as given:

$$
\dot{m}_{g}=\sum_{i}^{n} N N_{i} c_{f} \frac{P_{\text {Upstrean }}}{\sqrt{T_{\text {Upstream }}}}(\mathrm{kg} / \mathrm{s})
$$

Where $P_{\text {Upstream }}$ and $T_{\text {Upstream }}$ represent the absolute upstream pressure and absolute upstream temperature respectively, $N N_{i}$ represents the nozzle number for each of the nozzles in the nozzle bank and has been determined experimentally in relation to its cross-sectional area and the discharge coefficient of the nozzle, and $c_{f}$ is a constant with a value of $0.001 \mathrm{~K}^{1 / 2} \mathrm{~ms} P a / b a r$.

However, using the gauge pressure at the transport line and the temperature measured upstream of the nozzle bank, the gas flow at the transport line can be estimated during each of the experimental test run since the pressure acting on the sand particles in the transport line is assumed to be in the absolute unit, then Equation (4) can be used to calculate the gas flow velocity.

$$
V_{\text {gas }}=\frac{\dot{m}_{g}}{\frac{\left(P_{\text {guage }}+1.01325 \text { bar }\right)}{R T} \cdot A}
$$

\section{SIGNAL PROCESSING}

Several signal processing techniques were employed to extract relevant features from the acoustic signal in order to study the association between the sand characteristics data in the flowline and the acoustic emissions. The following section presents a summary of feature extraction techniques.

\subsection{Structure for feature extraction techniques and Machine learning models}

The acoustic signals have inherently nonstationary characteristics, however, an assumption is made that on short-time scales the signals are stationary. Based on this assumption, the feature extraction process was decomposed to form a structure for sequential mathematical operations that would lead to a more compact computation. The first step is pre processing of the acoustic signal by decomposing into a small number of frames duration of $10-30 \mathrm{~ms}$ in sequence, next a hamming windowing function is applied to reduce spectral distortions due to discontinuity at the edges of the frame [16-17].Signal transforms are used to map the representation of the signal from one domain to another. The goal is to transform the earliest interpretation of the signal into a shape that could be more practical for feature extraction processes[2]. The signal transforms used in this work are presented as follows: The Discrete Fourier Transform (DFT) is an increasingly important concept in digital signal processing applications including frequency analysis of signals and it is used to provide frequency domain representations of signals [18-19]. The Hilbert transform provides the instantaneous frequency energy distributions with time localities [20]. The Discrete Cosine Transform (DCT) is yet another promising technique widely and extensively used in signal processing applications due to its non-complex nature and the coefficients are real [18-19].

Aggregation can give a better description of feature dynamics. Aggregation of features using simple summary statistics can capture features that describe the characteristics of the signal which can be seen as an important element in the future reduction procedure and can result in a feature vector that is compact and complete with high discriminant power [21-22].. Both the time and frequency domains were considered in the feature extraction process: Short-Term Energy: the energy of a signal is by definition describes its ability to perform work. It is assumed that the energy of the acoustic signal gives information about the interaction of the sand particles with the flowline bend [23-24]. The Zero Crossing Rate (ZCR) is a weighted crossings within the frame in the time domain i.e the number of times the signal changes form within the frame, hence ZCR can also be regarded as a metric of uncertainty [2][17]. The Energy Entropy represents a feature useful to capture rapid transformations in the energy level of a signal as a consequence of sand particles/inner wall/gas collisions at the flowline bend[2]. Practically, the Shannon entropy 
[25] is used on the sequence in a bid to describe the uncertainty of the frame signal energy. Notionally the energy entropy can be elucidated as a measure of unpredictability within a system [2][26]. The Spectral Centroid of an acoustic signal defines the nexus where most of the energy in the frequency division is accumulated. On the other hand the Spectral Spread describes a metric about the form of the spectrum. In another sense, the spectral spread is a metric that is used to define whether the spectrum is accumulated in the vicinity of its centroid or it is simply distributed over the spectrum[17][21]. The spectral flux is a basic measure for rapid detection of transformations in the signal spectral structure overtime. In essence, the spectral flux captures the fluatuation of the spectrum between two adjacent frames in the signal. The spectral Entropy (SE) is an attribute useful in the detection of the degree of uncertainty in the signal spectrum. Moreover, It is also a metric that defines the peakiness or otherwise the flatness of the spectrum [27-28]. Spectral Rolloff (SR) is simply an attribute that is extensively used in a variety of machine learning applications. As an illustration, Marko [29] apply the spectral rolloff in a bid to distinguish speech acoustic signal [6]. Mel-Frequency Cepstral Coefficients (MFCC) algorithm is one of the standard techniques for acoustic characterization and is widely used in various domains including bioacoustics identification[30] and ASR [31]. The coherency of this standardized algorithm allows the efficient calculation of several cepstral coefficients which are usually taken as features. Additionally, it is perceptually inspired algorithm that mimics the hearing characteristics of the human ear [32]. The resulting coefficients are used to represent the spectral energy distribution of the signal

\subsection{Artificial Neural Networks (ANNs)}

Notionally the ANNs are considered as computational models that take inspiration from the biological neurons and have been developed since the seminal neural model of McCulloch and Pitts[2][33]. ANNs are commonly used in applications ranging from concept learning to function approximation. Structurally, there exist interconnections between the neurons and these interconnections have weights associated them and each neuron in the network performs a simple computation and application of an activation function. This generates an input to any of the participant neuron in the succeeding layer [34]. The weights are initially randomised, the process of weight modification is called learning and the method of learning used in this work is called supervised learning. It is supervised because the network is presented with samples that have inputs and outputs. It is supervised because the network is presented with samples that have inputs [features] and outputs [labels] during the learning phase. The prediction models were implemented through the application of TDNN. By contrast with classical multi-layered ANN structure, TDNN has a delay line tapped at the input. A TDNN has its input neurons modified by introducing a delay line. Such neurons will now have their inputs multiplied by several weights, one for each delay and one for the undelayed input. However, following this modification, the model can be used to detect the dynamics of the modelled process [35-36]. In this work a three layer TDNN has been modelled and is depicted in Figure 3. As shown in Figure 3, it has one hidden layer of ten neurons. Each neuron in the hidden layer has hyperbolic tangent function as the nonlinear activation function and the output neuron has a pure linear activation function. The hyperbolic tangent function was chosen as the activation of the hidden layer simply because of it posses excellent mathematical characteristics. The structure of the model is thus $2 L 10 \mathrm{~N} 1 \mathrm{~L}$, where $L$ represents linear processing neuron and $\mathrm{N}$ indicates nonlinear processing neurons. This TDNN structure is applied to model the non-linear temporal dependencies in the acoustic feature vector pattern for the estimation and prediction of the gas velocity and sand concentration[6].

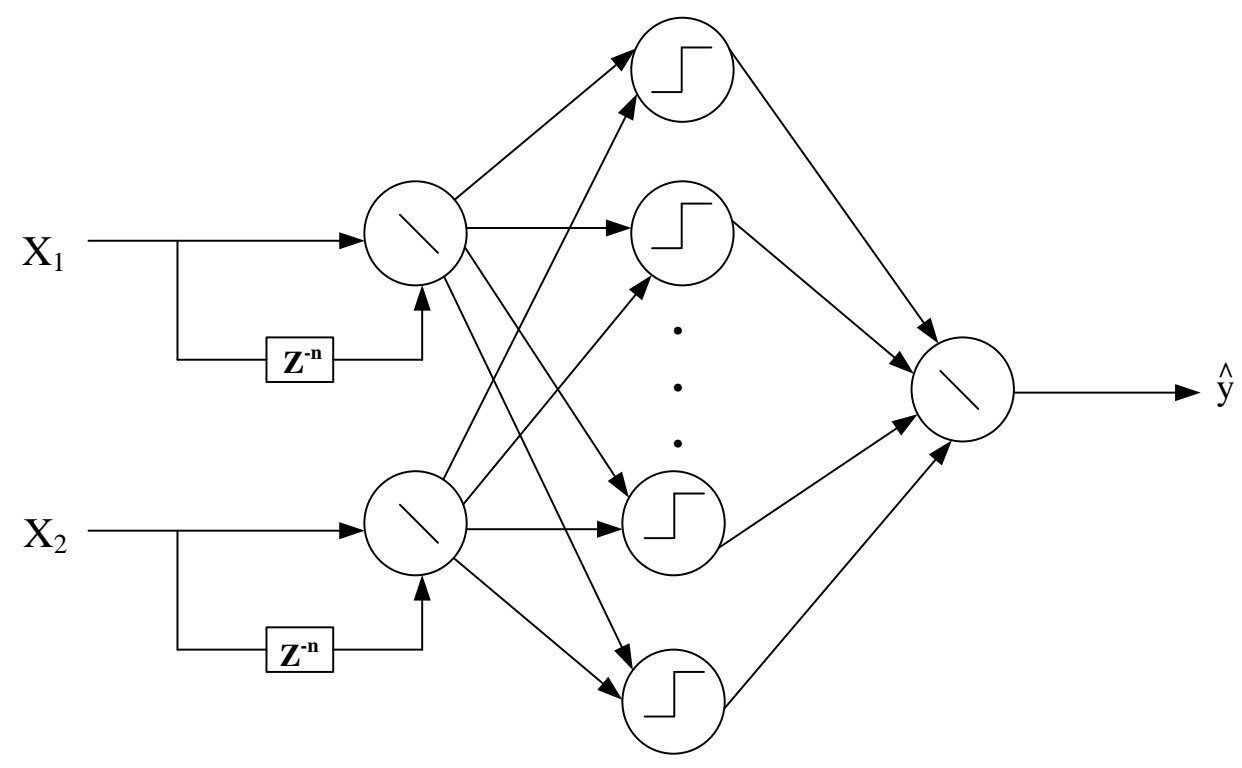

Figure 3 - A three layer TDNN 
International Journal of Advances in Scientific Research and Engineering (ijasre), Vol 5 (12), December-2019

\section{RESULT AND DISCUSSION}

An extensive analysis was undertaken of the signal processing and machine learning techniques, however, only one example is presented. Results from neighbourhood component analysis, NCA, identify nine (9) features as the most relevant to form the feature vector, which for illustration, two features, spectral centroid and spectral entropy were selected to build the prediction models presented in this paper. For simplicity of analysis, five instances of target event belonging to five (5) different sand particles' concentration acoustics were considered from the test database and are shown in Table 1.

Table 1 - Database used for the signal processing experiment

\begin{tabular}{|l|c|l|c|c|c|}
\hline SI. No & $\dot{\boldsymbol{m}}_{\boldsymbol{g}}(\mathbf{k g} / \mathbf{s})$ & $\mathbf{d p}(\mathbf{b a r})$ & $\dot{\boldsymbol{m}}_{\boldsymbol{s}}(\mathbf{k g} / \mathbf{s})$ & Sand Concentration $(-)[\mathbf{S C}]$ & $\boldsymbol{V}_{\boldsymbol{g a s}}(\mathbf{m} / \mathbf{s})$ \\
\hline $\mathbf{1}$ & 0.0552 & 1.085 & 2.409 & 44 & 10 \\
\hline $\mathbf{2}$ & 0.0623 & 1.062 & 2.207 & 35 & 11 \\
\hline $\mathbf{3}$ & 0.064 & 0.989 & 1.847 & 29 & 12 \\
\hline $\mathbf{4}$ & 0.0551 & 0.441 & 0.637 & 12 & 14 \\
\hline $\mathbf{5}$ & 0.0653 & 0.208 & 0.198 & 3 & 18 \\
\hline
\end{tabular}

Figure 4 and Figure 5 depict the histogram of the mean value of sequences of the selected attributes (spectral centroid and spectral entropy) derived from the original signal. As shown in Figure 4 the spectral centroid for the SC = 3.0, 11.6 and 43.7 are noticed to exhibit high values. This shows that the spectrum of the signals for those sand concentrations is essentially controlled by high frequencies. However, the mere sudden aspect of the results is the higher spectral centroid value for the SC $=43.7$ signal as the high frequency band indicates suspension of the sand particles in the medium. This could be due to the fact that the flow mechanism for that sand concentration was in quasi-suspension flow. In contrast the respective values for this statistic are relatively lower for the other signals. Notwithstanding, taking the uncertainty of particle-laden gas flow behaviours into consideration, these findings are therefore significant[6].

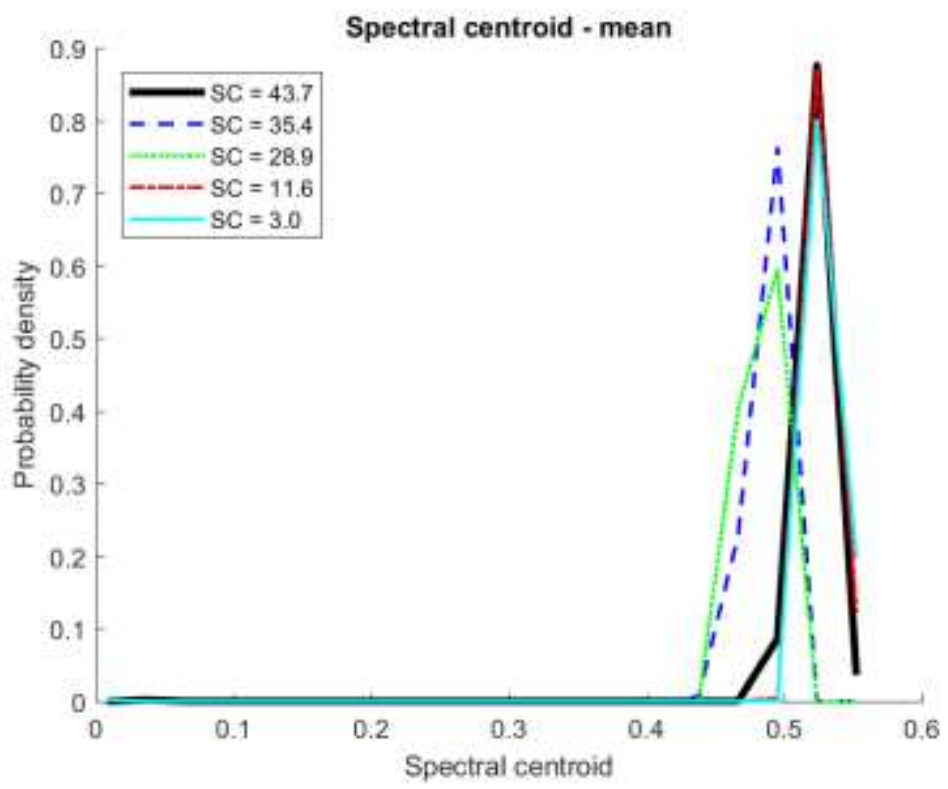

Figure 4 - Histogram of the mean of the spectral spread segments of the acoustic signals for different sand concentrations

Spectral entropy gives an approach to detect the complicacy of a signal and invariant transformation in the signal spectrum. The results for the histogram of the mean of the sequences for spectral entropy segments from the five signals considered for analysis as given in Figure 4 indicates the variation of probability density as a function of the spectral entropy values, it is however difficult to elucidate on the behaviours in terms of sand particles collision with the flowline bend. Broadly speaking, the spectral entropy value is low for a spectrum with flat distribution whereas spectra which contain sharp peaks exhibit higher values. It can be seen from Figure 5 that the $\mathrm{SC}=3.0$ and $\mathrm{SC}=43.7$ signal give the lowest spectral entropy value amongst the different sand particle concentrations. This is obvious because there are uncertainties in the distribution of energy in the signal spectra. Seemingly, these values for this statistic for the spectral entropy of SC $=35.4$ and SC $=28.9$ stay almost identical to that of SC $=$ 11.6 returning a relatively high spectral entropy value and thus implying small variations in the energy distribution. Similarly, the 
pattern for $\mathrm{SC}=43.7$ and $\mathrm{SC}=3.0$ remain the same but at relatively lower spectral value. In general, these high values in the spectral entropy provide an indication for the degree of randomness in the spectra of the signals[6].

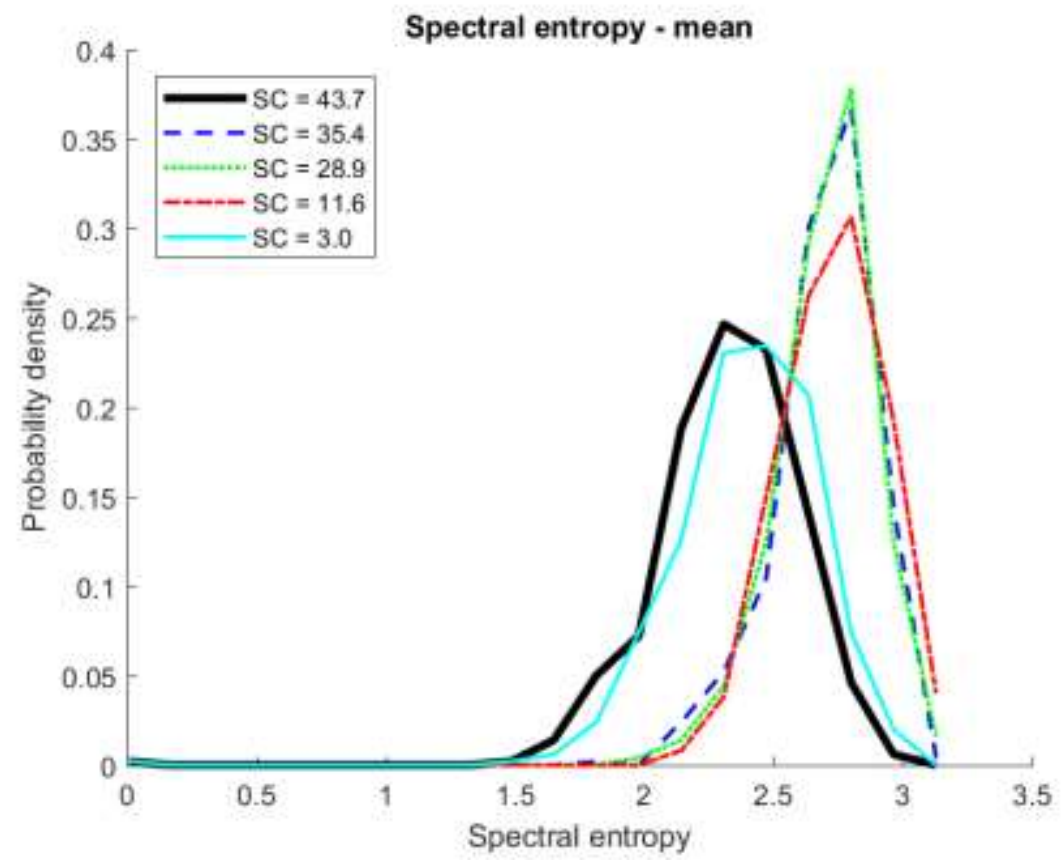

Figure 5 - Histogram of the mean of the spectral entropy segments of the acoustic signals for different sand concentrations

Additionally the results of the performance evaluation for the recognition of the sand characteristics data in the flowline are presented in terms of identification accuracy described by the NRMSE.

Table 2 - Performance of TDNN method for sand concentration and gas velocity models

\begin{tabular}{|l|l|l|}
\hline & Sand Concentration (-) & $\boldsymbol{V}_{\text {gas }}(\mathbf{m} / \mathbf{s})$ \\
\hline NRMSE & 0.17 & 0.16 \\
\hline
\end{tabular}

In order to test the efficiency of the model with the selected features, the results are presented in terms of the NRMSE and are tabulated in Table 2. These results indicate that the TDNN model has potential for the gas solid flow behaviour recognition based on acoustic signal measurement at a bend in a multiphase flowline.

\section{CONCLUSION}

The combination of the signal processing techniques and the machine learning approach has offered a powerful method for online quantitative monitoring of solid particulate materials in a gas flowline. In addition the signal processing methods are critical in this approach and provide a methodology which allows the acoustic data to be reduced into such a form that the machine learning method can easily work with. These methods forming the basis of the feature extraction are computationally efficient. Other than the practical methodology, the most important conceptual innovation of the present research is the consideration for Time-Delay Neural Networks (TDNN) - a variation of classical neural networks prepared to accommodate dynamics in flow mechanism, as the predictive model. The TDNN model has the ability to learn complex nonlinear decision surface. The performance of the TDNN over testing data from the solid characteristics in the flowline has been extensively evaluated. Results obtained demonstrate that with the classical ANN, the error NRMSE is 0.29 and 0.46 for the sand concentration, and gas velocity respectively. With the TDNN model, the NRMSE is 0.17 and 0.16 for the sand concentration and gas velocity respectively. In comparison with the ANN model, the TDNN model has better performance as the NRMSE values are lower for all the models for the measurands. The power of the TDNN lies in their ability to develop shift invariance for making optimal decisions. This could go a long way in helping to overcome the representational weakness of other conventional recognition systems faced with the uncertainty and variability in real-world data.

\section{REFERENCE}

B. Dudley, “BP Energy Outlook 2017 edition BP Energy Outlook 2017 edition,” 2017.

[2] K. T. Aminu, D. McGlinchey, and A. Cowell, "Acoustic signal processing with robust machine learning algorithm for 
improved monitoring of particulate solid materials in a gas flowline," Flow Meas. Instrum., vol. 65, 2019.

[3] T. Haugsdal, "The Most Efficient Use of Acoustic Sand Monitors . Lessons Learned from Many Years of Operation Sand Monitoring Acoustic Sand Monitoring Introduction,” Soc. Pet. Eng., no. SPE-185888-MS, pp. 1-12, 2017.

[4] M. E. El-Alej, "Monitoring Sand Particle Concentration in Multiphase Flow Using Acoustic Emission Technology," World Acad. Sci. Eng. Technol., vol. 7, no. 6, pp. 1-7, 2014.

[5] M. Odigie, S. A. Shirazi, B. S. McLaury, and S. Cremaschi, "Acoustic Monitor Threshold Limits for Sand Detection in Multiphase Flow Production System,” SPE Int. Conf. Exhib. Oilf. Corros., vol. 154378, pp. 1-13, 2012.

[6] K. T. Aminu, D. Mcglinchey, and Y. Chen, "Journal of Petroleum Science and Engineering Optimal design for real-time quantitative monitoring of sand in gas fl owline using computational intelligence assisted design framework," J. Pet. Sci. Eng., vol. 177, no. May 2018, pp. 1059-1071, 2019.

[7] M. E. El-Alej, M. Corsar, and D. Mba, "Monitoring the presence of water and water-sand droplets in a horizontal pipe with Acoustic Emission technology,” Appl. Acoust., vol. 82, pp. 38-44, 2014.

[8] D. Mba, T. Yan, and M. Elforgani, "Monitoring Sand Transport Characteristics in Multiphase Flow in Horizontal Pipelines Using Acoustic Emission Technology,” vol. 7, no. 6, pp. 892-898, 2013.

[9] Y. Hu, X. Qian, X. Huang, L. Gao, and Y. Yan, "Online continuous measurement of the size distribution of pneumatically conveyed particles by acoustic emission methods," Flow Meas. Instrum., pp. 1-6, 2014.

[10] M. Guo, Y. Yan, Y. Hu, D. Sun, X. Qian, and X. Han, "On-line measurement of the size distribution of particles in a gas solid two-phase fl ow through acoustic sensing and advanced signal analysis," Flow Meas. Instrum., pp. 1-9, 2014.

[11] Y. Hu, L. Wang, X. Huang, X. Qian, L. Gao, and Y. Yan, "On-line Sizing of Pneumatically Conveyed Particles Through Acoustic Emission Detection and Signal Analysis," pp. 1-10, 2014.

[12] J. Sun and Y. Yan, "Non-intrusive characterisation of particle cluster behaviours in a riser through electrostatic and vibration sensing," Chem. Eng. J., vol. 323, pp. 381-395, 2017.

[13] D. Sun, Y. Yan, R. M. Carter, G. Lu, G. Riley, and M. Wood, "Detecting the Presence of Large Biomass Particles in Pneumatic Conveying Pipelines Using an Acoustic Sensor,” pp. 3-6.

[14] K. Wang et al., "Acoustic sensor approaches for sand detection in sand - water two-phase fl ows," Powder Technol., vol. 320, pp. 739-747, 2017.

[15] M. G. Droubi, R. L. Reuben, and J. I. Steel, "Flow noise identification using acoustic emission ( AE ) energy decomposition for sand monitoring in fl ow pipeline," Appl. Acoust., vol. 131, pp. 5-15, 2018.

[16] D. Gerhard, “Audio Signal Classification : History and Current Techniques,” 2003.

[17] T. Giannakopoulos, Introduction to AUDIO ANALYSIS : A MATLAB Approach. 2014.

[18] J. G. Proakis and D. G. Manolakis, Digital Signal Processing: Principles, Algorithms, and Applications, Fourth. New Jersey: Pearson Education, 2007.

[19] T. K. Rawat, "Discrete Fourier Transform," in Digital Signal Processing, Oxford University Press.

[20] N. E. Huang et al., "The empirical mode decomposition and the Hilbert spectrum for nonlinear and non-stationary time series analysis," Proc. R. Soc. A Math. Phys. Eng. Sci., vol. 454, no. 1971, pp. 903-995, 1998.

[21] D. Mitrović, M. Zeppelzauer, and C. Breiteneder, "Features for Content-Based Audio Retrieval," in Advances in Computers, vol. 78, no. 10, 2010, pp. 71-150.

[22] P. Ruvolo, I. Fasel, and J. R. Movellan, "A learning approach to hierarchical feature selection and aggregation for audio classification,” Pattern Recognit. Lett., vol. 31, no. 12, pp. 1535-1542, 2010.

[23] R. C. Guido, “A tutorial on signal energy and its applications,” Neurocomputing, vol. 179, pp. 264-282, 2016. 
[24] D. Sun et al., "On-line nonintrusive detection of wood pellets in pneumatic conveying pipelines using vibration and acoustic sensors,” IEEE Trans. Instrum. Meas., vol. 63, no. 5, pp. 993-1001, 2014.

[25] C. E. Shannon, “A Mathematical Theory of Communication,” Bell Syst. Tech. J., vol. 27, no. 3, pp. 379-423, 1948.

[26] A. Mittal, S. S. Mallick, and P. W. Wypych, "An investigation into pressure fluctuations for fluidized dense-phase pneumatic transport of fine powders," Powder Technol., vol. 277, pp. 163-170, 2015.

[27] M. García, J. Poza, D. Abásolo, D. Santamarta, and R. Hornero, "Analysis of intracranial pressure signals recorded during infusion studies using the spectral entropy," in Conference proceedings : ... Annual International Conference of the IEEE Engineering in Medicine and Biology Society. IEEE Engineering in Medicine and Biology Society. Annual Conference, 2013, vol. 2013, pp. 2543-2546.

[28] L. K. Isaacson, "Spectral entropy, empirical entropy and empirical exergy for deterministic boundary-layer structures," Entropy, vol. 15, no. 10, pp. 4134-4158, 2013.

[29] M. Kos, Z. Kačič, and D. Vlaj, "Acoustic classification and segmentation using modified spectral roll-off and variancebased features,” Digit. Signal Process. A Rev. J., vol. 23, no. 2, pp. 659-674, 2013.

[30] T. L. F. Evangelista, T. M. Priolli, C. N. S. Jr, B. a. Angelico, and C. a. a. Kaestner, "Automatic Segmentation of Audio Signals for Bird Species Identification,” 2014 IEEE Int. Symp. Multimed., pp. 223-228, 2014.

[31] M. Sahidullah and G. Saha, "Design, analysis and experimental evaluation of block based transformation in MFCC computation for speaker recognition," Speech Commun., vol. 54, no. 4, pp. 543-565, 2012.

[32] J. X. Du, C. M. Zhai, Y. L. Guo, Y. Y. Tang, and P. C. Chun Lung, "Recognizing complex events in real movies by combining audio and video features," Neurocomputing, vol. 137, pp. 85-95, 2014.

[33] W. S. McCulloch and W. Pitts, “A logical calculus of the ideas immanent in nervous activity," Bull. Math. Biophys., vol. 5, no. 4, pp. 115-133, 1943.

[34] M. Riedmiller, "Advanced supervised learning in multi-layer perceptrons - from backpropagation to adaptive learning algorithms," Comput. Stand. Interfaces, vol. 16, no. 3, pp. 265-278, 1994.

[35] J. Wang, I. Tsapakis, and C. Zhong, “A space - time delay neural network model for travel time prediction,” Eng. Appl. Artif. Intell., vol. 52, pp. 145-160, 2016.

[36] A. Waibel, T. Hanazawa, G. Hinton, K. Shikano, and J. K. Lang, "Phoneme Recognition Using Time-Delay Neural Networks," IEEE TRANSANCTIONS Acoust. SPEECH, SIGNAL Process., vol. 37, no. 3, pp. 1-12, 1989. 